\title{
Négocier la mise en récit du passé. I'histoire de la fondation allemande « Mémoire, responsabilité, avenir » au prisme des théories de la reconnaissance
}

Le 17 décembre 1999 à Berlin, un accord historique est conclu. Le gouvernement allemand et les représentants des grandes entreprises nationales décident

L'auteure : Joëlle HECKER est docteure de l'Institut d'études politiques (Paris). de financer la création d'une fondation, baptisée « Mémoire, responsabilité, avenir ", destinée à indemniser les victimes de travail forcé sous le nazisme. Ce jour-là, Johannes Rau, président fédéral allemand, déclare : " tous ceux qui soutiennent cette fondation, l'État et les entreprises, reconnaissent la responsabilité commune et le devoir moral qui découle des crimes qui ont été commis "'. D'emblée, il est question de reconnaissance. Il en va de même du texte officiel du traité, signé quelques mois plus tard, en juillet 2000, dont le préambule s'ouvre sur cette idée de reconnaissance ${ }^{2}$. Mais dans quelle mesure ce vocabulaire correspond-il à la réalité des négociations? Le lexique de la reconnaissance est-il un simple vernis ou décrit-il au contraire véritablement les mesures prises alors?

Cet articles a pour but d'explorer les rapports éventuels entre la réalité empirique de ce programme de réparations et les théories de la reconnaissance. Ma définition de la reconnaissance s'adosse surtout aux travaux d'Axel HONNETH. J'emprunte à son œuvre la définition de la reconnaissance comme un processus qui aboutit à un décentrement identitaire ${ }^{3}$. Reconnaître, c'est donc ultimement ménager une place à autrui dans la construction de sa propre identité, ce processus pouvant également avoir lieu entre des collectifs. ${ }^{4}$ Selon HonNeTH, ce

\footnotetext{
' Discours de Johannes RAU, 17.12.1999, in JANSEN Michael et SAATHOFF Günter, Gemeinsame Verantwortung und moralische Pflicht. Abschlussbericht zu den Auszahlungsprogrammen der Stiftung "Erinnerung, Verantwortung und Zukunft », Göttingen, Wallstein, 2007, p. 175.

2 Gesetz zur Errichtung einer Stiftung "Erinnerung, Verantwortung und Zukuft ", 2 août 200o. Ibid., p. 176. 3 Honneth Axel, "Unsichtbarkeit. Über die moralische Epistemologie von Anerkennung' ", in idem, Unsichtbarkeit. Stationen einer Theorie der Intersubjektivität, Frankfurt am Main, Suhrkamp, 2003, p. 22.

${ }^{4}$ HONNETH Axel, "Anerkennung zwischen Staaten. Zum moralischen Untergrund zwischenstaatlicher Beziehungen ", in idem, Das Ich im Wir. Studien zur Anerkennungstheorie, Berlin, Suhrkamp, 2010, p. 192.
} 
décentrement est toujours le produit d'une lutte ${ }^{5}$. Dès lors, les victimes d'injustices seraient les seules à même d'enclencher ce processus de décentrement, si toutefois elles entrent en lutte pour être reconnues ${ }^{6}$. Mais cette reconnaissance n'est pas faite que de mots : elle s'appuie nécessairement sur un substrat matériel?.

Cet article adopte une lecture chronologique mais non moins analytique des faits. Pour lier le cas des réparations à ces théories, il importera en effet de s'interroger d'abord sur les éléments qui permettent lier ces réparations à une lutte pour la reconnaissance. II s'agira donc d'identifier dans un premier temps les parties en présence, leurs motivations ainsi que le caractère conflictuel de leurs rapports. Puis il faudra se demander ensuite de quelle façon le récit du passé a été reformulé par la mise en place de ce programme d'indemnisations. Cette reformulation sera analysée en deux temps: nous parlerons d'abord des évolutions induites par le programme de réparations en lui-même, et ensuite de la mise en récit à l'œuvre au sein du fonds "Avenir » créé dans le cadre de cette fondation.

Bien que les événements relatés ici soient récents, il est possible de recourir à de nombreux documents d'archives. En effet, la fondation "Mémoire, responsabilité, avenir » a estimé nécessaire de rendre ces documents accessibles au public dès le programme d'indemnisation achevé, en 2007, et a enjoint les organisations partenaires à faire de même. À Berlin, dans les locaux de la fondation, nous avons consulté les papiers d'Otto GRAF LAMBSDORFF, qui permettent de retracer une grande partie de l'histoire des négociations, puisque cet homme politique du parti libéral prit représenta le gouvernement allemand sur ces questions de 1999 à 2001 . Nous nous sommes également penchés sur un grand nombre de documents qui couvrent la période de mise en application du traité de 1999 à 2007. On trouve enfin dans ce fonds d'archives de précieux témoignages provenant des questionnaires remplis par les victimes quelques mois après leur indemnisation.

En ce qui concerne la littérature, de nombreux ouvrages ont d'ores et déjà été consacrés à l'histoire de cette fondation. Un grand nombre de juristes et d'historiens se sont penchés sur la question. Il faut mentionner en particulier le projet international dirigé par Constantin

\footnotetext{
5 Honneth Axel, "Die soziale Dynamik von Mißachtung. Zur Ortsbestimmung einer kritischen Gesellschaftstheorie ", in idem, Das andere der Gerechtigkeit. Aufsätze zur praktischen Philosophie, Frankfurt am Main. Suhrkamp, 2000, pp. 88-109.

6 Ibid.

7 Honneth Axel, «Anerkennung als Ideologie », WestEnd, 1, 2004, pp. 51-70.
} 
GOSCHLER publié en $2012^{8}$. En 2014 est parue également la monographie de Henning BORGGRÄFE, qui relate cette histoire en se concentrant sur les institutions allemandes 9 . La question des réparations a peu intéressé les politistes jusqu'ici. On trouve certes l'étude de Anja HENSE, mais celle-ci n'évoque pas la question de la reconnaissance ${ }^{10}$. Si l'on peut donc estimer que l'historiographie a déjà fait son travail, ce n'est pas encore le cas de la science politique en général, et encore moins de la théorie politique en particulier.

\section{Ia genèse de la Fondation « Mémoire, Responsabilité, Avenir $॥$, une lutte pour la reconnaissance ?}

La genèse de ce programme de réparations connut deux phases. La première est celle des procès en class action aux États-Unis, qui poussèrent le nouveau chancelier allemand Gerhard SCHRÖDER à soutenir la création d'une fondation destinée à régler cette question en janvier 1999. Cette phase est essentiellement germano-américaine. Ensuite, il y eut les négociations multilatérales qui visèrent à définir les modalités de fonctionnement de cette fondation jusqu'en juillet 2000 . Cette phase a vu l'entrée en scène des gouvernements d'Europe de l'Est et de certaines ONG.

\section{Les factures non réglées de la Seconde Guerre mondiale}

Si les crimes dont il est question ici ont été perpétrés en Europe, c'est pourtant aux États-Unis que se trouve la source du programme d'indemnisations lancé en 1999. Certes, les historiens allemands se penchèrent sur la question du travail forcé dès la fin des années 1970, mais leurs découvertes n'eurent dans un premier temps aucun impact du point de vue de l'indemnisation des victimes : tout au plus parvinrent-ils à faire en sorte que cette question fusse soulevée au Bundestag." Les acteurs de premier plan furent d'abord les cabinets d'avocats représentants les victimes, qui à la fin des années 1990 intentèrent des procès en class actions contre des firmes multinationales allemandes accusées d'avoir exploité des travailleurs forcés pendant

\footnotetext{
8 Goschler Constantin (dir.), Die Entschädigung von NS-Zwangsarbeit am Anfang des 21. Jahrhunderts, Göttingen, Wallstein, 2012, 1143 p.

9 BoRggräFE Henning, Zwangsarbeiterentschädigung. Vom Streit um "vergessene Opfer "zur Selbstaussöhnung der Deutschen, Göttingen, Wallstein, 2014, 562 p.

10 Hense Anja, Die Entstehung der Stiftung "Erinnerung, Verantwortung und Zukunft "für die Opfer von NS-Zwangsarbeit und Arisierung, Münster, Westfäliches Dampfboot, 2008, 384 p.

" BORGgRÄFE Henning, Zwangsarbeiterentschädigung, op. cit., pp. 150-170.
} 
la guerre ${ }^{12}$. En fait, avant de s'intéresser aux firmes allemandes, ces mêmes cabinets avaient attaqué des banques suisses, ce qui avait mené à la publication des rapports BERGIER et VOLCKER et donné lieu à un règlement financier entre les banques helvètes et les cabinets d'avocats ${ }^{13}$. Le succès engrangé par ces derniers les incita à poursuivre leur action ${ }^{14}$. Un élément décisif fut en outre l'attitude de l'administration CLINTON ${ }^{15}$. Ces procès eurent en effet une résonance telle que le gouvernement américain décida de s'impliquer en nommant un représentant, Stuart EIZENSTAT, chargé dès 1997 de négocier avec les banques suisses. Il devint par la suite l'interlocuteur des firmes allemandes sur la question de l'indemnisation du travail forcé ${ }^{16}$. Cet ancien membre de l'administration CARTER lança dans ce contexte l'idée d'une "solution globale " qui permettrait aux victimes d'être indemnisées et aux entreprises de ne plus faire face à ce type de plaintes ${ }^{17}$.

En Allemagne, les grandes entreprises décidèrent elles aussi de s'organiser pour faire face à ce risque financier, tandis que Gerhard SCHRÖDER, leader du SPD et candidat à la chancellerie, promit lors de la campagne des législatives de créer une fondation pour régler cette épineuse question dans le cas où il serait élu ${ }^{18}$. Le but affiché par le candidat était double : il voulait contribuer à une action morale ${ }^{19}$, tout en soutenant les entreprises allemandes dans leur quête de paix juridique ${ }^{20}$. Peu après les élections de septembre 1998, la coalition gouvernementale constituée par le SPD et les Verts fit des propositions concrètes quant à la forme que devrait prendre cette fondation censée mêler fonds publics et contributions des entreprises. ${ }^{21}$ Le 16

\footnotetext{
12 Niethammer Lutz, "Von der Zwangsarbeit im dritten Reich zur Stiftung 'Erinnerung, Verantwortung und Zukunft'. Eine Vorgeschichte », in JANSEN Michael et SAATHOFf Günter, op. cit., Göttingen, Wallstein, 2007, p. 44.

13 Gribetz Judah et Reig Shari C., "The Swiss Banks Holocaust Settlement », in Ferstman Carla, Goetz Mariana et Stephens Alan. (dir.), Reparations for Victims of Genocide, War Crimes and Crimes against Humanity. Systems in Place and Systems in the Making, Leiden, Boston, Martinus Nijhoff, 2009, pp. 115-142.

14 BORGgRÄFE Henning, Zwangsarbeiterenschädigung, op. cit., p. 246.

15 Eizenstat Stuart E., Imperfect Justice. Looted Assets, Slave Labor, and the Unfinished Business of World War II, New York, Public Affairs, 2003, p. 4.

16 SWIFT Robert A., "Holocaust Litigation and Human Rights Jurisprudence ", in BAZYLER Michael J. et Alford Roger p. (dir.), Holocaust Restitution. Perspectives on the Litigation and its Legacy, New York et Londres, New York University Press, 2006, p. 53.

17 Niethammer Lutz, "Von der Zwangsarbeit im dritten Reich zur Stiftung 'Erinnerung, Verantwortung,Zukunft' », op. cit., p. 44.

18 Ibid.

19 Ibid.

${ }^{20}$ Ibid., p. 46.

${ }^{21}$ Ibid., pp. 48-49.
} 
février 1999, treize grandes entreprises allemandes prirent à leur tour la parole et entérinèrent la création de la fondation "Mémoire, histoire, avenir $n^{22}$. II restait à définir les rôles dévolus à chacun et bien entendu la somme qui serait reversée aux victimes.

\section{Victimes et coupables}

Peut-on véritablement parler de lutte pour la reconnaissance dans un tel contexte? Tout d'abord, sur la forme, on peut constater qu'on a bien affaire à une lutte au cours de laquelle s'affrontent deux parties. Distinguer le groupe des coupables de celui des victimes est pourtant moins aisé qu'il n'y paraît. À première vue, on a d'un côté les entreprises et le gouvernement allemand, de l'autre les victimes résidant aux États-Unis, leurs avocats et le gouvernement américain soucieux de représenter leurs intérêts. Mais certains activistes allemands, surtout au sein du parti écologiste, avaient dès les années 1980 œuvré en faveur d'une indemnisation du travail forcé. Or, ce parti était représenté dans la nouvelle coalition gouvernementale et put de ce fait participer aux négociations de 1999, en prenant souvent des positions opposées à celles du monde économique, parfois même à celle du SPD. On peut évoquer l'action de Günter SAATHOFF, qui s'occupait depuis le milieu des années 1980 de la question des «victimes oubliées " par les programmes de réparations allemands et qui participa activement aux négociations. Il deviendra plus tard directeur de la fondation "Mémoire, responsabilité, avenir "23. Cet exemple prouve qu'il serait faux d'opposer deux camps, les victimes luttant pour obtenir réparation d'un côté, les Allemands opposés aux paiements de l'autre, même si le caractère conflictuel de ces négociations ne fait aucun doute: deux camps se sont bien affrontés, mais des Allemands jouèrent un rôle crucial de part et d'autre.

\section{Reconnaissance et intérêts}

Qu'en est-il de l'objet de la lutte? D'après HonNETH, la lutte pour la reconnaissance est le fruit de l'injustice, qu'il définit en termes de mépris et d'humiliation : autrement dit, les individus ou les groupes n'entrent pas en lutte pour des principes abstraits, mais parce qu'ils

\footnotetext{
22 Niethammer Lutz, "Von der Zwangsarbeit im dritten Reich zur Stiftung 'Erinnerung, Verantwortung,Zukunft' », p. 51. Ce chiffre évolua au fil du temps: cf. SPILIOTIs Susanne-Sophia, "Corporate Responsibility and Historical Injustice ", in KeANE John Horacio, Civil Society. Berlin Perspectives, New York, Berghahn, 2006, p. 65. 
éprouvent un sentiment d'injustice ${ }^{24}$. L'expérience du mépris, quand elle est partagée, peut donc donner lieu à des luttes collectives ${ }^{25}$. Dans le cas qui nous occupe, la voix des victimes est officiellement relayée par les cabinets d'avocats, le gouvernement américain et certains activistes allemands. Ceci pose la question des motifs : dans quelle mesure ces avocats, ce gouvernement et ces activistes se sont-ils faits le relais de demandes de reconnaissance? Ne poursuivent-ils pas des buts purement mercantiles en s'investissant de la sorte? C'est un argument que n'hésitèrent d'ailleurs pas à employer certaines entreprises allemandes à l'époque ${ }^{26}$, pourtant elles aussi engagées pour des motifs financiers avant tout. Comment en revanche interpréter l'attitude du gouvernement américain ou celle des activistes allemands? Selon Stuart EIZENSTAT, c'est bien un idéal moral qui a mu l'administration CLINTON à ce moment de l'histoire, même s'il ne nie pas les enjeux de politique étrangère ${ }^{27}$. II est certain en tous les cas que la rhétorique des droits de l'homme joua un rôle crucial pour ce gouvernement ${ }^{28}$. Sans doute, tous les acteurs impliqués dans ce plan de réparations n'étaient pas épris d'un désir de justice ${ }^{29}$. Certains songeaient à leurs intérêts financiers ou souhaitaient la paix juridique. On peut aussi supposer qu'au sein d'un même groupe d'acteurs, des motifs parfois contradictoires cohabitèrent. Mais l'intérêt de ces groupes convergea vers la mise en place de ce programme en faveur des victimes. Dès lors, plutôt que de lire des gestes selon un critère qui distinguerait les gestes moraux purs des bas intérêts, il semble plus juste de considérer que les intérêts se définissent pour tout collectif aussi bien de façon pragmatique que normative ${ }^{30}$. Comme le note HONNETH, cela s'applique également aux États, qui ne sont pas que des monstres froids : les décisions politiques sont prises en fonction des besoins institutionnels et des attentes normatives des citoyens ${ }^{31}$. Dans toute lutte pour la reconnaissance, les relais institutionnels sont une absolue nécessité. S'ils peuvent par leur intervention donner l'impression que la morale cède la place à l'intérêt, eux seuls sont à même de convertir

\footnotetext{
24 Honneth Axel, « Die soziale Dynamik von Mißachtung », op. cit., p. 99.

${ }_{25}$ HONNETH Axel, Kampf um Anerkennung. Zur moralischen Grammatik sozialer Konflikte, Frankfurt am Main,

Suhrkamp, 1992, p. 260.

${ }^{26}$ BorggräFE Henning, Zwangsarbeiterenschädigung, op. cit., p. 378.

${ }^{27}$ EIzenSTAT Stuart E, Imperfect Justice, op. cit., p. 5.

${ }^{28}$ BORgGrÄFE Henning, Zwangsarbeiterentschädigung, op. cit., p. 305.

29 KENT Roman, "It's Not about the Money. A Survivor's Perspective on the German Foundation Initiative", in BAZYLer Michael J. et Alford Roger p. (dir.), Holocaust Restitution. Perspectives on the Litigation and its Legacy, New York et Londres, New York University Press, 2006, p. 206.

30 Honneth Axel, Der Kampf um Anerkennung, op. cit., p. 266.

31 Honneth Axel, "Anerkennung zwischen Staaten », op. cit., p. 192.
} 
une attente normative en action politique significative ${ }^{32}$. À ce stade, ces relais institutionnels étaient les cabinets d'avocats des victimes et les États-Unis d'une part, les entreprises et le gouvernement allemand d'autre part. Dans un deuxième temps, la situation devint plus complexe, et les demandes de reconnaissance ne s'adressèrent plus exclusivement à l'Allemagne.

\section{La concurrence des victimes}

Après les annonces faites en février 1999, les États-Unis et l'Allemagne décidèrent de convier à la table des négociations des gouvernements d'Europe de l'Est ainsi que des organisations représentatives des victimes ${ }^{33}$. Finalement, se joignirent la Pologne, I'Ukraine, la Biélorussie, la Russie, la République Tchèque, Israël et une ONG, la Claims Conference. L'Américain EIZENSTAT suggéra de procéder selon le schéma suivant : chaque organisation partenaire devrait négocier son propre plafond, en fonction du nombre de victimes dont elle défendait les intérêts et de la nature des persécutions subies. Cette somme-plafond serait prélevée sur la somme globale versée par la fondation, dont le montant restait à fixer ${ }^{34}$. On s'arrêta ensuite sur dix milliards de Deutsche Mark, le gouvernement et les entreprises assumant chacun pour moitié le financement du fonds ${ }^{35}$. II restait pour les organisations représentatives des victimes à se partager cette somme. Un tel contexte était naturellement propice à l'émergence d'un phénomène de "concurrence des victimes », expression forgée par JeanMichel CHAUMONT ${ }^{36}$ et reprise abondamment dans l'historiographie des réparations ${ }^{37}$.

Cette concurrence se nourrissait en particulier d'une question, qui était celle de la distinction entre les travailleurs forcés et les esclaves, les seconds devant bénéficier d'une somme plus élevée ${ }^{38}$. La distinction avait déjà été faite dans le cadre de l'accord entre les cabinets d'avocats et les banques suisses en 1998, et EIZENSTAT avait dès mars

\footnotetext{
32 HECKER Joëlle, Les temps et les modes de la reconnaissance. La RFA, Israël et la Claims Conference 19501990, Thèse de doctorat soutenue à l'IEP de Paris, février 2014, p. 141.

33 Niethammer Lutz, "Von der Zwangsarbeit im dritten Reich zur Stiftung 'Erinnerung,

Verantwortung,Zukunft' », op. cit., p. 48.

34 BORGGRÄFE Henning, Zwangsarbeiterentschädigung, op. cit., p. 359.

35 Niethammer LutZ, «Von der Zwangsarbeit im dritten Reich... », op. cit., p. 61.

${ }^{36}$ Chaumont Jean-Michel, La concurrence des victimes. Génocide, identité, reconnaissance, Paris, La Découverte, 1997, $392 \mathrm{p}$.

37 Niethammer LutZ, "Von der Zwangsarbeit im dritten Reich... », op. cit., p. 62; BorgGräFE Henning, Zwangsarbeiterentschädigung, op. cit., p. 374 .

${ }^{38}$ Niethammer Lutz, « Von der Zwangsarbeit im dritten Reich... », op. cit., p. 62.
} 
1999 annoncé que l'une des conditions posées par les États-Unis en vue de la conclusion d'un accord était le maintien de ces deux catégories $^{39}$. La catégorie des esclaves désignait ceux qui avaient travaillé dans les camps de la mort. Elle concernait presque uniquement les victimes juives ${ }^{40}$. La Claims Conference veillait donc à maintenir cette distinction, estimant qu'il serait juste que les esclaves obtiennent des sommes trois fois supérieures aux autres ${ }^{41}$. Les pays d'Europe de l'Est cherchèrent quant à eux à réduire le différentiel : les esclaves devaient selon eux toucher le double des autres tout au plus ${ }^{42}$. Finalement, la proposition de la Claims Conference fut acceptée, les gouvernements d'Europe de l'Est estimant la somme de 5.000 Deutsche Mark obtenue par les travailleurs forcés satisfaisante ${ }^{43}$. II n'en reste pas moins que cet épisode avait démontré que les victimes étaient loin de former un groupe homogène et menant un combat commun contre l'Allemagne. Bien au contraire, rancœurs et ressentiments caractérisaient les liens entre ces groupes, qui luttaient donc aussi entre eux pour la reconnaissance.

Ainsi, il y a bien eu luttes pour la reconnaissance, mais ce terme doit être employé au pluriel, puisque durant la seconde phase des négociations, celle de la concurrence des organisations partenaires, les demandes de reconnaissance ne furent pas univoques.

\section{Le programme d'indemnisations, un geste de reconnaissance?}

Si l'on compare ce programme de réparations aux lois d'indemnisation antérieures, un certain nombre d'évolutions semblent aller dans le sens d'une plus grande reconnaissance, même si persistent beaucoup d'obstacles. La reconnaissance, selon la définition de HONNETH dont nous nous inspirons, n'est pas faite uniquement de mots. Au contraire, un substrat matériel lui est indispensable. Dans le même temps, ces mots comptent beaucoup. Telle est l'ambiguïté consubstantielle à la notion de reconnaissance. Au regard de l'histoire des réparations allemandes depuis 1952, qu'est-ce que ce programme apporte concrètement dans ce long parcours de la reconnaissance?

\footnotetext{
39 BORGGRÄFE Henning, Zwangsarbeiterentschädigung, op. cit., p. 314.

40 Niethammer Lutz, "Von der Zwangsarbeit im dritten Reich... », op. cit., p. 62.

4l Ibid.

42 Ibid.

43 Ibid.
} 


\section{La définition des destinataires}

Qui a été reconnu comme victime par ce nouveau programme de réparation ? Et qui a véritablement bénéficié de ces paiements? Ces deux questions doivent être distinguées, en raison du caractère tardif de ce programme.

\section{La fin du principe de territorialité}

Le programme de réparations de la fondation « Mémoire, responsabilité, avenir " n'inclut, pas plus que les programmes de réparations précédents, toutes les catégories de victimes. Bien au contraire, le $\$ 11$ de la loi voté par le parlement allemand exclut explicitement certains groupes de victimes ${ }^{44}$. Les prisonniers de guerre, notamment, furent écartés ${ }^{45}$. En outre, chaque organisation partenaire était en droit de proposer un ordre de priorité dans les indemnisations, de sorte que les travailleurs agricoles notamment ne purent bénéficier du programme d'indemnisation en Russie, son gouvernement ayant mal négocié son plafond ${ }^{46}$.

Le programme de 2000 se distinguait néanmoins des lois de réparations qui avaient été votées depuis 1952 par la République fédérale allemande, dans la mesure où la discrimination que subissaient les victimes originaires d'Europe de l'Est prit fin ${ }^{47}$. La fondation "Mémoire, responsabilité, avenir " entérina l'abandon du principe de territorialité ${ }^{48}$. Ainsi, jusque-là, les victimes originaires du bloc de l'Est avaient été exclues du bénéfice des lois d'indemnisation. Les conditions pour bénéficier des pensions au titre de la loi BEG (Bundesentschädigungsgesetz) votée 1953 notamment étaient très restrictives: seules les victimes ayant vécu dans les frontières du Reich

\footnotetext{
${ }_{44}$ Gesetz zur Errichtung einer Stiftung "Erinnerung, Verantwortung und Zukunft ", 2.8.2OOO, in JANSEN Michael et SAATHOFF Günter, "Gemeinsame Verantwortung und moralische Pflicht ». Abschlussbericht zu den Auszahlungsprogrammen der Stiftung "Erinnerung, Verantwortung und Zukunft », op. cit., p. 184.

45 Aujourd'hui, alors que seuls 4.000 anciens prisonniers de guerre vivent encore, le gouvernement allemand a finalement décidé de les indemniser. cf. "Deutschland entschädigt sowjetische Kriegsgefangene ", Die Zeit, 20.05.2015.

${ }^{46}$ Penter Tanja, «Zwischen Misstrauen, Marginalität und Missverständnissen - Zwangsarbeiterentschädigung in Russland, Litauen und Lettland ", in GosCHLER, Constantin (dir.), Die Entschädigung von NS-Zwangsarbeit am Anfang des 21. Jahrhunderts. Helden, Opfer, Ostarbeiter - das Auszahlungsprogramm in der ehemaligen Sowjetunion, Göttingen, Wallstein, 2012, p. 256.

47 Goschler Constantin, Schuld und Schulden. Die Politik der Wiedergutmachung für NS-Verfolgte seit 1945 , Göttingen, Wallstein, 2005, p. 470. 
de 1937 ou de langue maternelle allemande étaient éligibles ${ }^{49}$. Avec la création de la fondation "Mémoire, responsabilité, avenir ", ces barrières tombèrent, puisque seul le type de persécution subie servait désormais de critère d'éligibilité.

\section{Un groupe décimé}

Mais l'extension du groupe des bénéficiaires survint longtemps après la fin de la guerre, alors même que le nombre de survivants diminuait rapidement. Au moment des négociations de 1999, les chiffres avaient de quoi donner le vertige. Stuart EIZENSTAT enjoignit les Allemands à prendre des décisions rapides, $10 \%$ des victimes concernées mourant chaque année ${ }^{50}$. Un représentant des victimes polonaises avançait quant à lui le chiffre de $20 \%{ }^{51}$. C'est ce que l'historien Raul TEITELBAUM appelle cyniquement la "solution biologique » au problème des réparations, qu'il accuse les gouvernements allemands successifs de mettre en œuvre ${ }^{52}$. II est vrai que les négociations furent bien plus longues que prévues, si bien que les reproches se multiplièrent. Pour ne pas donner l'impression de jouer la montre, les Allemands déclarèrent donc que la date qui serait inscrite dans la loi serait celle de l'annonce de l'initiative, soit le 16 février 1999 : ainsi, les héritiers de toute personne décédée après cette date aurait droit à une indemnisation, moins élevée cependant que celle des premiers concernés ${ }^{53}$.

La signification de ces paiements fut en tout état de cause grandement amoindrie par leur caractère tardif. Pour bien des survivants, la crainte de ne jamais percevoir la somme prévue était grande et la signification symbolique de ces paiements s'en voyait nécessairement entachée ${ }^{54}$. Finalement, parmi les victimes qui avaient vécu jusqu'à la date butoir du 15 février 1999, 20\% décédèrent durant la phase des

\footnotetext{
49 BRUNNER José et NACHUM Iris, " 'Vor dem Gesetz steht ein Türhüter'. Wie und warum israelische Antragsteller ihre Zugehörigkeit zum deutschen Sprach- und Kulturkreis beweisen mussten ", in BRUNNER José, FreI Norbert et Goschler Constantin, Die Praxis der Wiedergutmachung. Politik, Moral, Moralpolitik, Göttingen, Wallstein, 2010, pp. 387-424.

5o Authers John et Wolffe Richard, The Victim's Fortune: Inside the Epic Battle Over the Debts of the Holocaust, New York, Harper Collins, 2002, p. 214.

51 BoRgGrÄFE Henning, Zwangsarbeiterschädigung, op. cit., p. 313.

52 Teitelbaum Raul, Die biologische Lösung. Wie die Shoah wiedergutgemacht wurde, Berlin, Klampen, 2008, $366 \mathrm{p}$.

53 AUthers John, "Making Good Again. German Compensation for Slaved and Forced Laborers ", in DE GrEIFF Pablo, The Handbook of Reparations, Oxford, Oxford University Press, 2006, p. 435.

54 Fondation "Mémoire, responsabilité, avenir » (Berlin), Classeurs "Prüfung Ex Post », JCC, 24.3.2004; 25.3.2004; 26.3.2004; 21.6.2004.
} 
paiements ${ }^{55}$. Finalement, si la définition des destinataires a été considérablement élargie, ce groupe était, au moment des paiements, bien plus restreint qu'il ne l'aurait été au sortir de la guerre. La fin du principe de territorialité, inscrite dans la loi et traduite dans les faits, eut donc une portée restreinte.

\section{L'attribution des responsabilités}

Qui a reconnu ses responsabilités ? Et peut-on véritablement parler d'assomption de responsabilité ? Il est là encore difficile de conclure à un accomplissement de la reconnaissance.

\section{Une responsabilité socialisée}

La fondation "Mémoire, responsabilité, avenir » marque l'avènement d'une "responsabilité socialisée », comme le souligne l'historien Henning BORGGRÄFE ${ }^{56}$. Dans un registre optimiste, cette expression pourrait laisser à penser qu'une responsabilité collective est assumée, cette fois-ci non pas seulement par quelques dirigeants politiques, comme ce fut le cas dans l'Allemagne d'ADENAUER en 1952, mais également par de nombreux représentants de la société civile, à commencer par le monde économique. Les entreprises allemandes ayant pris part aux négociations avaient d'ailleurs annoncé que l'un de leurs deux objectifs, outre la paix juridique, était l'assomption d'une "responsabilité morale ${ }^{57}$. En outre, dans le préambule du traité, le lien entre ces paiements et le rôle des entreprises sous le nazisme est souligné, comme l'avaient souhaité certains députés du Bundestag ${ }^{58}$. En tout état de cause, dans la mesure où cette fondation est née de la coopération entre le gouvernement et le monde économique, et se fonde sur l'expertise d'activistes issus de la société civile, elle s'appuie davantage sur l'ensemble de la société que les plans de réparations précédents, conçus au ministère des finances et votées par le parlement.

Mais du point de vue de la reconnaissance, la responsabilité socialisée est à double tranchant. Ainsi, dans les faits, les contributions se sont faites sur la base du volontariat, sans aucune contrainte. Cela signifie que la responsabilité effective des entreprises, le fait qu'elles aient ou non exploité des travailleurs forcés durant la guerre, n'a absolument pas été prise en compte. Certes, les treize entreprises pré-

55 BORGGRÄFE Henning, Zwangsarbeiterentschädigung, op. cit., p. 455.

56 Ibid., p. 216 et suivantes.

57 Ibid., p. 405.

58 Ibid., p. 407. 
sentes dès le départ ${ }^{59}$ avaient toutes exploité des travailleurs forcés et ont finalement reversé $60 \%$ des cinq milliards prévus ${ }^{60}$. Mais le dispositif dans son ensemble et les campagnes lancées en direction de toutes les entreprises faisait fi de la réalité des responsabilités historiques et se fondait entièrement sur l'idée d'une société globalement responsable, et d'entreprises au devoir moral identique ${ }^{61}$. La responsabilité socialisée, c'est donc aussi celle qui repose sur tout le monde en faisant oublier la spécificité du rôle de chacun. Ceci n'est pas sans poser problème et rappelle l'une des remarques que fit Hannah ARENDT sur la dilution de la responsabilité dans l'Allemagne d'après-guerre : si tout le monde est également responsable, alors personne ne l'est ${ }^{62}$.

D'un point de pratique néanmoins, il faut préciser que plus de soixante-dix ans après les faits, il est difficile d'établir un lien entre les entreprises impliquées à l'époque et celles qui existent aujourd'hui, même si elles portent le même nom. La responsabilité socialisée serait dès lors une réponse, certes imparfaite, problématique, mais néanmoins efficace, à cette question épineuse de la responsabilité.

\section{Une responsabilité limitée}

Dans l'histoire des réparations, une thématique récurrente est celle du point final. À chaque fois qu'un gouvernement allemand s'engagea dans un programme de réparations, il espéra, il insista même souvent pour que l'autre partie s'engageât à ce que ses revendications fussent les dernières. Dès 1952, ADENAUER avait déclaré devant le Bundestag que le traité germano-israélien mettrait fin au chapitre le plus sombre de l'histoire allemande ${ }^{63}$. En 1965, quand la loi d'indemnisation BEG fut amendée, le chancelier ERHARD estima que l'aprèsguerre était terminé ${ }^{64}$. En 1980, lors de la création d'un nouveau fonds d'indemnisation, le gouvernement de Helmut SCHMIDT annonça à la Claims Conference que ce geste serait le dernier ${ }^{65}$. Pour sa part, le

\footnotetext{
59 Allianz, BASF, Bayer, BMW, DaimlerChrysler, Deutsche Bank, Degussa Hüls, Dresdner Bank, Höchst, Krupp, Siemens, Thyssen, Volkswagen.

60 SpILIOTIS Susanne-Sophia, Verantwortung und Rechtsfrieden. Die Stiftungsinitiative der deutschen Wirtschaft, Frankfurt am Main, Fischer, 2003, p. 198.

61 BORGgRäFE Henning, Zwangsarbeiterentschädigung, op. cit., p. 407.

62 ARENDT Hannah, "Was heißt persönliche Verantwortung unter einer Diktatur? ", in idem, Nach Auschwitz. Essays und Kommentare 1, Berlin, Tiamat, 1989, p. 81.

63 Discours d'Adenauer, Verhandlungen des Deutschen Bundestages, 4 mars 1953, vol. 15, lère période législative, session 252, p. 12092.

${ }^{64}$ Goschler Constantin, Schuld und Schulden, op. cit., p. 219.

65 Goschler Constantin, "Die Bundesrepublik und die Entschädigung von Ausländern seit 1966 ", in Hockerts Hans Günter, MoISEl Claudia et Winstel Tobias (dir.), Grenzen der Wiedergutmachung. Die Entschädigung für NS-Verfolgte in West und Osteuropa 1945-2000, Göttingen, Wallstein, 2006, p. 110.
} 
gouvernement de Helmut KoHL précisa au sujet d'un fonds d'indemnisation créé en décembre 1987 qu'il serait le dernier ${ }^{66}$. En 1999, lorsque les négociations commencèrent, la question du point final fut posée une nouvelle fois. Gerhard SCHRÖDER déclara souhaiter en cette fin de siècle refermer un chapitre ${ }^{67}$. II souligna même que toute revendication future serait rejetée ${ }^{68}$. La politique du passé de SCHRÖDER se situait donc la droite ligne des politiques de normalisation de ses prédécesseurs ${ }^{69}$. Il faut rappeler que c'est également pour parvenir à une solution définitive que les entreprises s'engagèrent dans ces négociations : leur objectif était d'obtenir la paix juridique. ${ }^{70}$ C'est pourquoi la dernière phase de discussions tourna entièrement autour de cette question. II fut finalement décidé que chaque personne indemnisée devrait signer un formulaire par lequel elle s'engagerait à ne plus jamais réclamer d'indemnisation à l'Allemagne. C'est la première fois que les victimes devaient s'engager personnellement à ne plus rien réclamer à l'Allemagne, et ce en dépit de leur âge avancé et alors même que leur nombre ne cessait de diminuer.

\section{Le médium de la réparation}

Comment ces gestes de reconnaissance ont-ils eu lieu ? Quelle a été leur nature et comment interpréter leur portée? Une fois de plus, l'ambiguïté est de mise.

\section{Un " geste humanitaire »}

Le ministre des finances fédéral Hans EICHEL ainsi que les avocats représentant les intérêts des entreprises firent tout pour que rien ne contraignît l'Allemagne à payer. C'est pourquoi les réparations furent d'emblée définies par ces acteurs comme un geste humanitaire ${ }^{71}$. Ils veillèrent à ce qu'il ne soit jamais question dans la loi ni de réparation ni de dédommagement. Parmi les nombreux exemples, on note une

\footnotetext{
66 Goschler Constantin, Schuld und Schulden, op. cit., p. 354.

${ }^{67}$ ARNING Matthias, Späte Abrechnung. Über Zwangsarbeiter, Schlußstriche und Berliner Verständigungen, Frankfurt am Main : Fischer, 2001, p. 14.

68 Fondation "Mémoire, responsabilité, avenir » (Berlin), papiers Lambsdorff, Termine und Gespräche, 13.9.1999.

${ }^{69}$ Schanetzky Tim, «Distanzierung, Verunsicherung, Entschädigung. Die deutsche Wirtschaft und die Globalisierung der Wiedergutmachung ", in OSTERLOH Jörg et WIXFORTH Harald (dir.), Unternehmer und NS-Verbrechen. Wirtschaftsleliten im 'Dritten Reich' und in der Bundesrepublik Deutschland, Frankfurt am Main, Campus, 2014, p. 148.

70 SpILIOTIS Susanne-Sophia, Verantwortung und Rechtsfrieden, op. cit., 314 p. 
intervention de la Deutsche Bank, qui rappelle qu'il faut à tout prix éviter le verbe to compensate et lui préférer to help dans les discussions avec Washington. ${ }^{72}$ Cela signifie que ces paiements furent effectués sur la base du volontariat et qu'aucune obligation légale ne fut reconnue. Leur signification s'en voit grandement relativisée. II faut souligner au passage que ce type de paiement dit "humanitaire " n'est pas nouveau, mais caractérise au contraire l'ensemble des plans de réparations allemands depuis 1965.

Günter SAATHOFF, l'actuel directeur de la fondation «Mémoire, responsabilité, avenir ", pense que la question de la terminologie est finalement secondaire : il va jusqu'à parler d'une « construction d'historiens ", sans rapport avec les attentes des victimes ${ }^{73}$. Selon lui, la seule chose qui comptait pour les victimes en 2000 , c'était de recevoir le plus rapidement possible la somme d'argent promise, que celle-ci porte le nom d'indemnisation ou pas ${ }^{74}$. Certains témoignages semblent aller dans ce sens, d'autant que pour les survivants du bloc de l'Est, les sommes versées étaient parfois, eu égard au niveau de vie dans ces pays, conséquentes ${ }^{75}$. Mais les survivants expriment souvent aussi de la déception face à des sommes perçues comme dérisoires si on les rapporte à la réalité de ce que furent ces travaux forcés ${ }^{76}$. II y aurait donc un forme de proportionnalité à respecter malgré tout.

Néanmoins, si on adopte un critère de proportionnalité, les réparations monétaires atteignent rapidement leur limite. De nombreuses victimes soulignent que l'argent ne saurait en aucun cas réparer les crimes commis, qui relèvent de l'irréparable $e^{77}$. Mais on peut estimer que l'argent est inadéquat tout en défendant le principe des réparations monétaires : on considère ces paiements avant tout comme des symboles qui manifestent l'existence d'une dette. C'est le cas de la Claims Conference, qui refusa d'ailleurs au nom de l'irréparabilité de signer un document qui rendrait impossible toute action contre le

\footnotetext{
${ }^{72}$ Fondation « Mémoire, responsabilité, avenir » (Berlin), papiers Lambsdorff, Termine und Gespräche, 21.10.1999.

73 Entretien avec Günter SAATHOFF, novembre 2015, Berlin.

74 Ibid.

75 AUthers John, " Making Good Again », op. cit., p. 435.

76 Fondation "Mémoire, responsabilité, avenir " (Berlin), Classeurs "Prüfung Ex-Post », JCC, 26.3.2004, 29.3.2004, 1.4.2004. 
gouvernement allemand à l'avenir. Cela aurait signifié selon elle mettre un point final à la question des réparations, et donner par là-même l'impression que l'argent avait suffi ${ }^{78}$.

\section{Dette et demande de pardon}

Si l'argent a le défaut de donner l'impression de réparabilité, c'est le mérite des réparations dites symboliques que de pouvoir faire un geste en direction des victimes tout en soulignant le caractère irréparable des crimes commis. Johannes RAU, le 17 décembre 1999, lors d'une cérémonie solennelle à l'issue de la première phase des négociations, fit un geste à portée symbolique. Dans un discours, il souligna le caractère irréparable des crimes commis et surtout demanda pardon aux victimes au nom du peuple allemand. Manifestement, ce geste faisait suite à une demande de la part des dirigeants de la Claims Conference ${ }^{79}$. Le discours de RAU s'acheva ainsi : "Je pense aujourd'hui à tout ceux qui eurent à subir sous le joug allemand des travaux forcés et furent réduits en esclavage et leur demande pardon au nom du peuple allemand. ${ }^{80}$. À bien des égards, il fait écho l'agenouillement de Willy BRANDT en 1970, qui s'apparentait à une demande de pardon silencieuse. Cette fois-ci, pour la première fois, la demande de pardon fut formulée explicitement, et ses termes négociés avec les victimes. Or, comme l'ont montré Jacques DERRIDA et Paul RIC氏UR, c'est bien dans le registre de l'irréparable que se joue le pardon : DERRIDA évoque une logique hyperbolique, RIC氏UR parle de surabondance ${ }^{81}$. Symboliquement, et parce qu'il s'accompagne de gestes concrets, la signification d'un tel discours est donc forte.

L'impact de cette demande de pardon est néanmoins difficilement mesurable. On peut noter l'émotion d'un survivant de la Shoah, Roman KENT présent lors de la cérémonie et qui prit la parole après le discours du président ${ }^{82}$. Celui-ci aurait interprété ce geste comme une victoire morale, bien plus significative que les paiements ${ }^{83}$. Le président du Congrès Juif Mondial de l'époque Israel SINGER dit en substance la même chose que KENT : le geste de RAU et la symbolique

\footnotetext{
78 TAYLOR Gideon, "Where Morality Meets Money ", in BAZYler Michael J. et Alford Roger p. (dir.), Holocaust Restitution. Perspectives on the Litigation and its Legacy, New York et Londres, New York University Press, 2006, p. 167.

79 Ibid., p. 166.

80 JANSEN Michael et SAATHOFF Günter, « Gemeinsame Verantwortung und moralische Pflicht », op. cit., p. 175.

${ }^{81}$ DerRIDA Jacques, Le siècle et le pardon. Entretien avec Michel Wievorka, Paris: Seuil, 2000, p. 111; RICEUR Paul, La Mémoire, l'histoire, l'oubli, Paris : Seuil, 2000, p. 625.

82 Authers John et Wolffe Richard, The Victim's Fortune, op. cit., p. 229.

${ }^{83}$ EIZENSTAT Stuart E, Imperfect Justice, op. cit., p. 3.
} 
qui l'entoure serait le principal acquis du mouvement en faveur des réparations dans les années $1990^{84}$. Mais Roman KENT et Israel SINGER étaient également des membres éminents de la Claims Conference, c'est-à-dire des personnes déjà acquises au principe des réparations. Il est néanmoins indéniable que ce geste marqua une rupture par rapport à la politique du passé menée par Helmut $\mathrm{KoHL},{ }^{85}$ qui sur le plan des symboles évitait les gestes de repentance. ${ }^{86}$ II faut toutefois souligner pour finir que si tout le monde en Allemagne se souvient du geste de BRANDT, ce n'est pas le cas du discours de RAU, qui n'a pas marqué la mémoire collective.

\section{Le Fonds u Avenir » : négocier une vision du passé}

Si le discours de RAU est presque oublié, le rôle de ce programme de réparations dans les politiques mémorielles en Allemagne est considérable. Une vision du passé fut négociée en 1999 et 2000 , et continue d'être négociée dans le cadre du fonds "Avenir » qui poursuit son action à ce jour.

\section{La réécriture du passé}

Par le biais de cette nouvelle loi de réparations, la politique mémorielle de l'Allemagne a évolué dans la mesure où, premièrement, des victimes oubliées ont enfin rejoint le cercle des bénéficiaires des réparations. En outre, l'existence même de cette fondation atteste du fait que les entreprises qui avaient exploité des travailleurs forcés durant la guerre ont bien commis un crime. Enfin, les sommes d'argent reversées matérialisèrent l'engagement en faveur des victimes

Doit-on donc en conclure qu'il y a bien eu décentrement identitaire en Allemagne, conformément à la définition de HonNETH ? Selon la psychanalyste Ilka QUINDEAU, très réservée à l'égard de ce concept de reconnaissance, il faudrait distinguer deux points. Selon elle, la mise en application de la loi, c'est-à-dire la façon concrète dont l'administration allemande a traité les demandes (et les victimes), démontre les limites

\footnotetext{
${ }^{84}$ BazYler Michael J., Holocaust Justice. The Battle for Restitution in America's Courts, New York: New York University Press, p. 300.

85 Wolfrum, Edgar, Rot-Grün an der Macht. Deutschland 1998-2005, Munich, Beck, 2013, p. 353.

${ }^{86}$ HERF Jeffrey, Zweierlei Erinnerung. Die NS-Vergangenheit im geteilten Deutschland, traduit de l'américain par Klaus Dieter SCHMIDT, Berlin, Propyläen, 1998, p. 414.
} 
de ce décentrement ${ }^{87}$. Si l'injustice des persécutions et du travail forcée a été sans conteste reconnue, ce n'est pas le cas des souffrances individuelles. Elle se fonde pour cela sur des entretiens menés avec des personnes indemnisées. C'est comme si au cours de ce processus réciproque, les Allemands avaient surtout cherché à redéfinir leur identité, sans faire le pas suivant qui les mènerait à reconnaître la souffrance des victimes ${ }^{88}$. La politique mémorielle allemande des années 2000 serait donc caractérisée par un certain égocentrisme, une forme de reconnaissance identitaire rapportée à soi plus qu'à l'autre.

Cela transparaît dans la manière dont le fonds "Avenir » fut constitué. Cela se fit en plusieurs phases. D'abord, durant les négociations, seuls les Allemands semblaient prêter attention à ce fonds, comme si la mémoire de la Shoah était devenue une affaire allemande. Ainsi, fin 1999, les entreprises, sur les 1,7 milliards de Deutsche Mark promis au départ, souhaitaient allouer $50 \%$ des sommes à ce fonds destiné à financer des projets de commémoration ${ }^{89}$. Elles se heurtèrent néanmoins rapidement à l'opposition de toutes les autres parties. Les avocats des victimes notamment insistèrent pour que $90 \%$ des fonds au moins reviennent aux travailleurs forcés. Ils proposèrent de doter ce fonds "Avenir" de 100 millions de Deutsche Mark seulement ${ }^{90}$. EIZENSTAT pensait également que les survivants devaient avoir la priorité, même s'il était prêt à concéder aux Allemands une somme plus élevée, soit environ 500 millions de Deutsche Mark ${ }^{91}$. LAMBSDORFF lui opposa que le fonds "Avenir » était la motivation principale des entreprises : ainsi, même les firmes créées après la guerre seraient incitées à participer ${ }^{92}$. Ce serait donc mettre en péril leur contribution que de réduire ce fonds à peau de chagrin ${ }^{93}$. Si les entreprises insistèrent tant pour mettre en valeur ce fonds, c'est qu'elles estimaient ce fonds comme très important pour leur image. En Allemagne, il n'y avait guère que les Verts pour penser tout comme EIZENSTAT que priorité devait être donnée à l'aide aux victimes encore en vie ${ }^{94}$. Finalement, sur les dix milliards que le gouvernement allemand et le monde économique

\footnotetext{
${ }^{87}$ QuINDEAU Ilka, "Erfahrungen jüdischer Antragsteller mit der Entschädigung für Zwangsarbeit », in Goschler Constantin, Brunner José, Ruchniewicz Krzysztof et Ther Philipp (dir.), Die Entschädigung von NS-Zwangsarbeit am Anfang des 21. Jahrhunderts. Band 2. Transnationale Opferanwaltschaft. Das Auszahlungsprogramm und die internationalen Organisationen, Göttingen, Wallstein, 2012, p. 253.

${ }_{88} \mathrm{Ibid}$.

89 BORGGRÄFE Henning, « Zwangsarbeiterentschädigung », op. cit., p. 355.

90 Ibid.

91 Ibid.

92 Fondation « Mémoire, responsabilité, avenir » (Berlin), papiers Lambsdorff, Termin und Gespräche, 26.1.2000..

93 lbid.

${ }^{94}$ BORGGRÄFE Henning, «Zwangsarbeiterentschädigung », op. cit., p. 355.
} 
s'engagèrent à payer, 700 millions furent attribuées à ce fonds. Mais avec ce fonds, il s'agissait plus pour les entreprises de se mettre en valeur plus que de tendre la main vers l'autre, ce qui confirme l'idée d'Ilka QUINDEAU : une redéfinition identitaire peut ressembler à une opération de décentrement tout en servant d'abord des intérêts propres.

\section{La question de l'avenir}

S'il est donc impossible de dire que les victimes ont été reconnues par ce programme de réparations, on peut en revanche constater que la question de la redéfinition ou du décentrement identitaire ne cesse pas d'être posée en 2000. Bien au contraire, avec la création de la fondation "Mémoire, responsabilité, avenir ", les représentants des coupables et des victimes décidèrent même d'institutionnaliser les discussions à ce sujet. Le fonds "Avenir » en particulier, qui devait dans un premier temps servir les intérêts des entreprises allemandes, s'avère avec le recul l'un des points les plus innovants du plan de réparations.

Aujourd'hui, alors que le programme des paiements est achevé, le conseil d'administration de la fondation est toujours composée des parties présentes lors des négociations, et tous décident de l'usage du fond "Avenir ». Les représentants des victimes veillent à ce que l'essentiel des fonds aille, aujourd'hui encore, au soutien aux survivants. II n'en reste pas moins qu'au fil des années, le nombre de survivants diminuant, les pôles " Histoire " et "Droits de l'homme ", qui existent depuis 2004, gagnent progressivement en importance. Mais dans ces domaines également, c'est un dialogue institutionnalisé entre les parties en présence qui dessine les contours de la politique mémorielle de la fondation. Finalement, le fonds "Avenir " réussit le tour de force de perpétuer le dialogue entre représentants des victimes et représentants des coupables au sujet de la forme que doit prendre le souvenir. L'idée de point final est ici clairement rejetée, et surtout, l'ouverture au point de vue de l'autre institutionnalisée. Du point de vue la reconnaissance, la symbolique du fonds "Avenir » est donc très forte et son action cruciale.

La juriste Leora BILSKY porte un jugement optimiste sur ce type de procédures, qui présentent selon elle l'avantage d'être ouvertes. En effet, plutôt que de décider des réparations dans l'enceinte de tribu- 
nal en prononçant une sentence, les leçons du passé sont négociées entre les parties, de nombreux représentants de la société civile prenant part au processus ${ }^{95}$. Elle rejoint en cela l'avis de l'historien Lutz Niethammer, qui note qu'à mesure que le temps passe, il s'agit de moins en moins de réparer ou de juger que de comprendre comment on a réparé ou pas: il s'agit d'écrire ensemble l'histoire de la mémoire ${ }^{96}$. De ce point de vue, le fond «Avenir » est un montage réussi.

\section{Conclusion}

Nous nous demandions en introduction si la fondation « Mémoire, responsabilité, avenir " pouvait être vue comme l'instrument d'une politique de reconnaissance, la reconnaissance étant ici définie comme une opération de décentrement. Autrement dit, le point de vue des victimes du nazisme a-t-il, oui ou non, été davantage intégré à la mémoire collective allemande par le biais de cette fondation?

Nous avons d'abord pu constater que dans ce programme de réparations, la logique de la charité a supplanté celle du droit : aucun droit à réparations n'est reconnu. Ainsi, ni l'État ni les entreprises ne reconnaissent leur responsabilité civile. En outre, cette reconnaissance est très tardive et les victimes sont bien souvent déçues des sommes dérisoires obtenues au regard des préjudices subis.

Pourtant, le travail forcé est sans conteste reconnu comme un crime par le gouvernement allemand, mais aussi par les entreprises, qui adoptent enfin la lecture de l'histoire proposée par bien des spécialistes depuis les années 1980. Du point de vue de la responsabilité collective, on constate également une évolution, des acteurs de la société civile étant pleinement intégrés au processus réparateur, là où les réparations étaient restées jusqu'alors l'apanage du politique. La fondation met également fin au principe de territorialité qui caractérisait la législation allemande depuis les années 1950 et avait été à l'origine de la discrimination des victimes du bloc de l'Est. Surtout, ce plan innove dans la mesure où il institutionnalise le dialogue entre les parties autour de la question du souvenir. En effet, le fonds "Avenir » peut être considéré comme un forum au sein duquel les organisations représentatives des victimes et les émissaires de la société civile et

95 BILSKY Leora, "Zwischen Washington und Berlin. Transnationale Holocaust-Entschädigung ", in BRUNNER José, Goschler Constantin et FreI Norbert, Die Globalisierung der Wiedergutmachung. Politik, Moral, Moralpolitik, op. cit., p. 227.

96 NiETHAMMER Lutz, "Wahrheitskommissionen im Vergleich. Haben wir bei der Zwangsarbeiterentschädigung den Wahrheitsauftrag verfehlt? ", in SEIDEL Hans-Christoph et TENFELDE Klaus, Zwangsarbeit im Europa des 20. Jahrhunderts. Bewältigung und vergleichende Aspekte, Essen, Klartext, 2007, p. 31. 
du gouvernement allemands s'interrogent jusqu'à aujourd'hui sur les contours que doit prendre la politique mémorielle de la fondation. II ne s'agit certes que d'un endroit, d'une institution au budget limité, mais elle semble constituer un modèle de ce à quoi devrait ressembler une mémoire décentrée du passé. 